\title{
EDITORIAL
}

\section{Is this the beginning of unravelling the puzzle of COPD exacerbations?}

\author{
R. Rodríguez-Roisin
}

$\mathbf{T}$ he natural history of chronic obstructive pulmonary disease (COPD) is one of a progressive lung function reduction, essentially expressed by an accelerated decline in forced expiratory volume in one second (FEV1), with limited exercise activity, poor health status and systemic (extrapulmonary) effects, all interspersed with a varying frequency of episodes of COPD exacerbation. Frequency of COPD exacerbations increases with increased disease severity, mostly in patients with advanced COPD (FEV1 <50\% predicted), who are often prone to frequent and repeated episodes.

COPD exacerbations have a serious impact on patients in terms of lung function decline or disease progression, morbidity and mortality, and poor quality of life, and involve huge economic costs. A background of lower FEV1 and frequent COPD exacerbations, defined as at least three episodes over the previous year within a geographic area [1], are considered vital risk factors for hospitalisation for a COPD exacerbation. Episodes of COPD exacerbation most commonly result in more severe airflow obstruction, with gas exchange [2] and pulmonary haemodynamic worsening [3], the latter being also predictive of hospitalisation for these exacerbations. Prognosis following severe hospitalised COPD exacerbation is also very poor, with high mortality rates at 2 yrs [4]. According to the World Health Organization, COPD deaths are set to increase to $\sim 5$ million annually, making this the fifth leading cause of global mortality [5]. Moreover, the socio-economic burden to the different healthcare services is dramatic. While a global survey estimated considerable annual direct costs [6], hospital admissions for COPD exacerbation increase the economic burden [7]. Altogether, COPD exacerbations represent one of the major battle fields of the physicians' clinical load, who in addition have access to a limited therapeutic armamentarium.

In the current issue of the European Respiratory Journal, new evidence of the complex interaction between dyspnoea severity and one of the abnormal components of lung mechanics, lung hyperinflation, during COPD exacerbations is provided by PARKER et al. [8]. Resting lung hyperinflation is a functional marker of COPD, particularly in advanced disease [9]. Acuteon-chronic lung hyperinflation is now considered one of the landmarks of COPD during exercise, which stays for several minutes. This study affords the proof of evidence of a similar

CORRESPONDENCE: R. Rodríguez-Roisin, Servei de Pneumologia, Hospital Clínic, Villarroel, 170, (IDIBAPS) Universitat de Barcelona, 08036-Barcelona, Spain. Fax: 34 932275404. E-mail: rororo@clinic.ub.es amount of acute-on-chronic lung hyperinflation during COPD exacerbations that is, however, sustained over several days.

Twenty patients with moderate-to-severe COPD exacerbation, mostly managed as outpatients, were followed sequentially within $72 \mathrm{~h}$ of their abrupt episodes for up to 2 months thereafter using symptom assessment (by means of clinical questionnaires), routine lung function tests, metabolic and breathing pattern measurements, and arterial oxygen saturation. There were three novelties. First, COPD exacerbations were associated with airflow obstruction and a worsening of lung hyperinflation, with marginal changes in the other remaining parameters. The lung volume recruitment secondary to the reduced air trapping represented the major contribution to increased airflow rates that, along with improvements in most lung volumes, were not accompanied with parallel changes in the FEV1/forced vital capacity (FVC) ratio. Secondly, breathlessness, the most constant symptom during COPD exacerbations, kept pace with forced spirometric (FEV1 and FVC) and lung volume (i.e. inspiratory capacity (IC), functional residual capacity and residual volume) improvements both at presentation and during follow-up. Symptomatic impairment-improvement was always accompanied with changes in the same direction of the principal functional outcomes. However, the bulk of recovery in dyspnoea and lung hyperinflation improved within 2 weeks of COPD exacerbation, slightly at variance with the median time of functional and symptomatic recovery of 6 days shown in a similar cohort of COPD exacerbation patients [10]. A third new finding was that the beneficial IC changes reliably reflected changes in end-expiratory lung volume, just to the extent that total lung capacity did not vary. The authors suggested that improvements in lung hyperinflation markers, such as increased IC, which occur in conjunction with dyspnoea reduction during recovery from COPD exacerbations, might become a useful functional descriptor of symptomatic amelioration. However, this contention, based on the finding that the observed IC changes are of the same magnitude of those shown in the resting IC response to acute short-acting bronchodilation in stable COPD [11], warrants further studies to be considered more robust. Unfortunately, a subsequent analysis of the potential interest of the subset of patients who clinically recovered against those that did not failed to provide firm conclusions.

The results of this study are of importance not only because they demonstrate that the common logistical difficulties to this type of designs can be successfully overcome, and the authors have to be commended for this remarkable achievement, but 
also because they can enrich the current understanding of the multidimensional heterogeneous pathophysiology of COPD exacerbations. By investigating lung hyperinflation response and its interplay with dyspnoea during COPD exacerbations, we can gain useful information on the factors that limit tidal volume expansion and induce increased loading and weakness of the inspiratory muscles in COPD. This dissociation between the neural drive to breathe and the mechanical response of the respiratory system may constitute, in turn, the basis of the clinical deterioration observed during exercise, a hypothesis previously suggested by O'DONNELL et al. [9].

One of the potential limitations of the study, already announced by the authors, was the nonstandardised therapy of COPD exacerbations that was given in accordance to the Canadian COPD guidelines. Patients were essentially treated under the premises of the "shotgun therapy", where most patients receive most available drugs, with varied dosages and durations of treatment. Notwithstanding, in my opinion, this should enhance the robustness of the findings as they are closer to the real world. The stringent criteria of randomised controlled trials (RCT) tend to include too many "special" patients, at variance with those attended in day-by-day clinical practice. In contrast, a weakness of the study not sufficiently addressed by the investigators was the modest gas exchange data provided. They argued that gas exchange defects were "small and less likely to contribute", akin with the moderateto-severe nature of COPD exacerbation, a view that needs to be in part tempered. During the first days of severe COPD exacerbations needing hospitalisation, worsening of gas exchange is primarily produced by increased alveolar ventilation to pulmonary blood flow imbalance, an effect that is amplified by a decreased mixed venous oxygen tension resulting from greater oxygen consumption [2]. Not unsurprisingly, in a RCT of systemic steroids in COPD exacerbation outpatients [12], with mean FEV1 values close to those shown in the current study, oxygen tension almost reached the borders of acute respiratory failure, hence reflecting the wide heterogeneity of COPD exacerbations. Unlike stable COPD, COPD exacerbations profoundly disturb pulmonary gas exchange and cardiovascular state. Moreover, a comprehensive assessment of the complex interplay between lung mechanics, gas exchange abnormalities and cardiovascular events is desperately needed in moderate-to-severe COPD exacerbations. This can be extremely helpful to improve the understanding of the multifactorial dimensions of acute COPD episodes and their relationships with the inflammatory response of the lung, whose pathogenic mechanisms still remain elusive. In addition to the increased presence of low alveolar ventilation to pulmonary blood flow regions, increased pulmonary hyperinflation during COPD exacerbations can contribute to the deterioration of alveolar ventilation to pulmonary blood flow inequalities by creating areas with high alveolar ventilation to pulmonary blood flow ratios [13]. It might be conceivable, therefore, that part of the differences among these three alluded studies [2, 8, 12] are inherently linked to the different criteria used for COPD exacerbations definition [14]. The latter was categorised as symptom-based [15] instead of being defined as event- or action-driven [16].

What are the implications of these findings? In my opinion, two critical aspects emerge. First, these results underline the importance of the heterogeneous nature of COPD exacerbations. While understanding of the nature and triggering factors of COPD exacerbations has increased enormously in recent years, these advances have also been useful to confirm and assess their complexity and their multidimensional origin, which vary enormously from patient to patient. This heterogeneity represents an additional burden to the difficult problem of COPD exacerbations, as an increase in the underlying inflammatory response of the lungs may not be common place to all abrupt episodes. A better knowledge of the different subsets of COPD exacerbation will not only provide deeper insights into their frequency, but also better definition and understanding may facilitate a tailored therapeutic approach and improve management.

Secondly, these findings set the stage for testing the beneficial effects of molecules, such as long-acting bronchodilators, known to efficaciously improve airflow obstruction, lung hyperinflation and air trapping in stable COPD $[17,18]$. The latter can be regarded as a very useful complementary medication to improve the current management of COPD exacerbations both prophylactically and therapeutically. We know that the improvements in exercise-related endurance time and dyspnoea post-long-acting bronchodilators are comparable with those achieved during exercise training [19]. There is much evidence that pulmonary rehabilitation improves both exertional and resting dyspnoea [20] and that its early use after admission for COPD exacerbations results in substantial exercise capacity and health status improvements at 3 months [21]. The combination of pharmacological and nonpharmacological approaches could be intelligently used during, and immediately after, COPD exacerbations to optimise multidimensionally the management and look for potential synergisms. The reduction of the deleterious symptomatic impact of increased lung hyperinflation will not only ameliorate breathlessness, but also can mutually improve alveolar ventilation to pulmonary blood flow imbalance and cardiovascular sequelae, hence collectively breaking the vicious cycle of more airflow obstruction, increased lung hyperinflation and air trapping, and further gas exchange worsening with cardiovascular complications, the most often scenario encompassing severe COPD exacerbations.

Early recognition of chronic obstructive pulmonary disease exacerbation symptoms and prompt institution of therapy by physicians improve the current tremendous burden of morbidity and mortality related to chronic obstructive pulmonary disease exacerbations [22]. Needless to say, this is key in our ongoing combat against chronic obstructive pulmonary disease.

\section{REFERENCES}

1 García-Aymerich J, Monsó E, Marrrades RM, et al. Risk factors for hospitalization for a chronic obstructive pulmonary disease exacerbation. EFRAM Study. Am J Respir Crit Care Med 2001; 164: 1002-1007.

2 Barberà JA, Roca J, Ferrer A, et al. Mechanisms of worsening gas exchange during acute exacerbations of chronic obstructive pulmonary disease. Eur Respir J 1997; 10: 1285-1291. 
3 Kessler R, Faller M, Fourgaut G, Mennecier B, Weitzenblum E. Predictive factors of hospitalization for acute exacerbation in a series of 64 patients with chronic obstructive pulmonary disease. Am J Respir Crit Care Med 1999; 159: 158-164.

4 Connors AF Jr, Dawson NV, Thomas C, et al. Outcomes following acute exacerbation of severe chronic obstructive pulmonary disease. Am J Respir Crit Care Med 1996; 144: 959-967.

5 World Health Organization. World Health Report 2002. Geneva: World Health Organization, 2002. www.who.int/ whr/2002/en/. Date accessed: 22 Oct 2003.

6 Wouters EF. Economic analysis of the Confronting COPD survey: an overview of results. Respir Med 2003; 97: Suppl. C, S3-S14.

7 Hernández C, Casas A, Escarrabill J, et al. Home hospitalization of exacerbated chronic obstructive pulmonary disease patients. Eur Respir J 2003; 21: 58-67.

8 Parker CM, Voduc N, Aaron D, Webb KA, O'Donnell DE. Physiologic changes during symptom recovery from moderate to severe exacerbations of COPD. Eur Respir J 2005; 25: 420-428.

9 O'Donnell DE, Webb KA. Exertional breathlessness in patients with chronic airflow limitation: the role of dynamic hyperinflation. Am Rev Respir Dis 1993; 148: 1351-1357.

10 Seemungal TAR, Donaldson GC, Bhowmik A, Jeffries DJ, Wedzicha JA. Time course and recovery of exacerbations in patients with chronic obstructive pulmonary disease. Am J Respir Crit Care Med 2000; 161: 1608-1613.

11 O'Donnell DE, Forkert L, Webb KA. Evaluation of bronchodilator responses in patients with "irreversible" emphysema. Eur Respir J 2001; 18: 914-920.

12 Thompson WH, Nielson CP, Carvalho P, Charan NB, Crowley JP. Controlled trial of oral prednisone in outpatients with chronic obstructive pulmonary disease. Am J Respir Crit Care Med 1996; 154: 407-412.

13 Rodriguez-Roisin R, Barberà JA, Roca J. Pulmonary gas exchange. In: Calverley PMA, MacNee W, Pride NB, Rennard SI, eds. Chronic Obstructive Pulmonary Disease 2nd Edn. London, Arnold, 2003; pp. 175-193.

14 Pauwels R, Calverley P, Buist SA, et al. COPD exacerbations: the importance of a standard definition. Respir Med 2004; 98: 99-107.

15 Rodriguez-Roisin R. Towards a consensus definition for COPD exacerbations. Chest 2000; 117: Suppl. 2, 398S-401S.

16 Vestbo J. What is an exacerbation of COPD. Eur Respir Rev 2004; 13: 88, 6-13.

17 O'Donnell DE, Voduc M, Fitzpatrick M, Webb K. Effects of salmeterol on the ventilatory response to exercise in chronic obstructive pulmonary disease. Eur Respir J 2004; 24: 86-94.

18 O'Donnell DE, Flüge $\mathrm{T}$, Gerken $\mathrm{F}$, et al. Effects of tiotropium on lung hyperinflation, dyspnoea and exercise tolerance in COPD. Eur Respir J 2004; 23: 832-840.

19 O'Donnell DE, McGuire M, Samis L, Webb K. The impact of exercise reconditioning on breathlessness in severe chronic airflow obstruction. Am J Respir Crit Care Med 1995; 152: 2005-2013.

20 Wouters EF. Management of COPD. Lancet 2004; 364: 883-895.

21 Man WD-C, Polkey MI, Donaldson N, Gray BJ, Moxham J. Community pulmonary rehabilitation after hospitalisation for acute exacerbations of chronic obstructive pulmonary disease: randomised controlled study. BMJ 2004; 329: 1209-1212.

22 Wilkinson TMA, Donaldson GC, Hurst JR, Seemungal TAR, Wedzicha JA. Early therapy improves outcomes of exacerbations of chronic obstructive pulmonary disease. Am J Respir Crit Care Med 2004; 169: 1298-1303. 